\title{
Intensive urate-lowering therapy improves structural joint damage in patients with tophaceous gout
}

A small exploratory study from Dalbeth et al. investigating the effect of pegloticase on radiographic damage in patients with tophaceous gout has shown that this treatment results in profound urate lowering and reduced joint bone erosion.

"Given our previous work showing that tophi and structural joint damage are very closely related in joints affected by gout, and recent clinical trials of pegloticase demonstrating rapid reduction in the size of tophi in those patients who responded to treatment, we wondered whether this treatment might also prevent progression of radiographic damage in people with tophaceous gout," explains Nicola Dalbeth, from the University of Auckland, New Zealand.

Radiographs of the feet and hands of eight patients with tophaceous gout were obtained at baseline and after 12 months of pegloticase treatment and were assessed using the gout-modified Sharp-van der Heijde score; serum urate

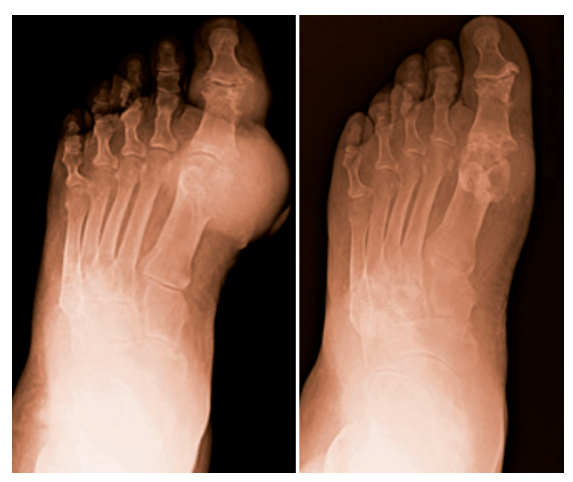

Radiographs at baseline (left) and after 30 infusions of pegloticase (right). Image courtesy of Herbert S. B. Baraf.

levels were also measured at these time points. All patients experienced extreme urate lowering (serum urate $<1 \mathrm{mg} / \mathrm{dl}$ ) and improvement in radiographic damage scores (median [range] reduced from 69.25 [1.5-138] at baseline to 57.25 [1.5-110] at 12 months). This improvement was due to reduced bone erosion scores (median [range] reduced from 44.0 [1.5-110] at baseline to 37.0 [1-71.5] at 12 months; $P=0.008$ ) and not to alterations in joint-space narrowing, which did not change (median [range] 28.25 [0-43] at baseline, 27.5 [0.5-41.5] at 12 months; $P=0.50)$.

"These findings suggest that in the context of profound urate lowering, bone remodelling in gout can occur, with healing of erosions," states Dalbeth. She continues: "For me, the key issue that arises from this work is whether such improvement in radiographic damage can occur with less intensive urate-lowering therapy, and if so, whether there is a specific serum urate target that is needed to achieve this target."

Jenny Buckland

Original article Dalbeth, N. et al. Exploratory study of radiographic change in patients with tophaceous gout treated with intensive urate-lowering therapy. Arthritis Care Res. doi:10.1002/acr.22059 